NOTAS 



\title{
LA POLÍTICA COMERCIAL COMÚN DE LA UNIÓN EUROPEA Y EL DESARROLLO SOSTENIBLE
}

\author{
ANA MANERO SALVADOR ${ }^{1}$ \\ Universidad Carlos III de Madrid \\ ana.manero@uc3m.es
}

Cómo citar/Citation

Manero Salvador, A. (2020).

La política comercial común de la Unión Europea y el desarrollo sostenible.

Revista de Derecho Comunitario Europeo, 66, 603-627.

doi: https://doi.org/10.18042/cepc/rdce.66.10

\section{Resumen}

El desarrollo sostenible es un objetivo para la Unión Europea en su acción exterior. La política comercial no ha sido ajena a la realización de este objetivo, de manera que ha desarrollado diferentes técnicas con el fin de implementarlo en sus relaciones con terceros Estados, como son la inclusión de los capítulos sobre desarrollo sostenible en los tratados de libre comercio, las evaluaciones de impacto o mecanismos preferenciales de carácter condicional. Asimismo, la Agenda 2030 establece un marco relacional de carácter finalista que tiene por objeto la consecución del desarrollo sostenible. Más concretamente, el ODS17 presta atención a cómo las relaciones comerciales deben tener en cuenta el desarrollo sostenible. Este trabajo analiza la adecuación de la labor desarrollada por la Unión Europea en este ámbito a la Agenda 2030, la conveniencia de los instrumentos establecidos y la naturaleza jurídica del desarrollo sostenible, tanto en el derecho internacional como en el ámbito de la Política Comercial Común.

\section{Palabras clave}

Desarrollo sostenible; Agenda 2030; tratados de libre comercio; SPG+; SIAs.

1 Profesora titular (acreditada como catedrática) de derecho internacional público. Universidad Carlos III de Madrid. Este trabajo es resultado del proyecto nacional de investigación "ODS, derechos humanos y derecho internacional» (PGC2018095805-B-I00). 


\section{THE EUROPEAN UNION COMMON COMMERCIAL POLICY AND SUSTAINABLE DEVELOPMENT}

\section{Abstract}

Sustainable development is a goal for the European Union in its external action. Trade policy has not been unconnected to the achievement of this objective, so it has developed different techniques in order to implement it in its relations with third States, such as the inclusion of the chapters on sustainable development in free trade agreements, impact assessments, or unilateral preferences. Likewise, the Sustainable Development Agenda establishes a relational framework that aims to achieve sustainable development. More specifically, SDG17 pays attention to how trade relations should take into account sustainable development. This paper analyzes the adequacy of the work carried out by the European Union in this area to the Sustainable Development Agenda, the convenience of the established instruments, and the legal nature of sustainable development both in International Law and in the field of the Common Commercial Policy.

\section{Keywords}

Sustainable development; 2030 Agenda for Sustainable Development; free trade agreements; GSP+; SIAs.

\section{LA POLITIQUE COMMERCIALE COMMUNE DE L'UNION EUROPÉENNE ET LE DÉVELOPPEMENT DURABLE}

\section{Résumé}

Le développement durable est un objectif de l'Union Européenne dans son action extérieure. La politique commerciale n'a pas été étrangère à la réalisation de cet objectif, elle a donc développé différentes techniques pour la mettre en œuvre dans ses relations avec les États tiers, comme l'inclusion des chapitres sur le développement durable dans les accords de libre-échange, évaluations d'impact ou mécanismes préférentiels conditionnels. De même, le Programme de développement durable à l'horizon 2030 établit un cadre relationnel qui vise à réaliser le développement durable. Plus précisément, l'ODD 17 s'intéresse à la manière dont les relations commerciales devraient prendre en compte le développement durable. Cet article analyse l'adéquation des travaux menés par l'Union européenne dans ce domaine du Programme 2030, les instruments développés et la nature juridique du développement durable tant en droit international qu'en matière de politique commerciale commune.

\section{Mots clés}

Développement durable; Programme de développement durable à l'horizon 2030; traités de libre-échange, SPG+; SIAs. 


\section{SUMARIO}

I. INTRODUCCIÓN. II. EL DESARROLLO SOSTENIBLE Y EL DERECHO INTERNACIONAL. APROXIMACIÓN A LAS RELACIONES COMERCIALES. III. LANATURALEZA JURÍDICA DEL DESARROLLO SOSTENIBLE EN LA POLÍTICA COMERCIAL COMÚN. IV. ¿CÓMO SE PREVÉ LA PROMOCIÓN DEL DESARROLLO SOSTENIBLE EN LA RELACIÓN COMERCIAL CON TERCEROS ESTADOS?: 1. Los capítulos sobre desarrollo sostenible. 2. Los SIAs. 3. EI SPG+. V. LA ADECUACIÓN DE LOS INSTRUMENTOS EUROPEOS A LA AGENDA 2030. VI. CONCLUSIÓN. BIBLIOGRAFIA.

\section{INTRODUCCIÓN}

La Unión Europea (UE) es uno de los principales actores comerciales de la comunidad internacional, junto con China y Estados Unidos. Una de las principales manifestaciones de su importancia económica es la celebración de tratados de libre comercio con terceros Estados. De hecho, la UE se caracteriza por el hecho de que su política comercial tiene alcance global, en la medida en que no se circunscribe a un área geográfica concreta, al tiempo que es la política comercial más ambiciosa del mundo ${ }^{2}$. Es más, esta política comercial es una de las principales manifestaciones de la acción exterior de la UE, y va a tener por finalidad «crear prosperidad y fomentar los valores comunes y universales que sustentan la UE» (Mäalstrom, 2016: 3), entre los que se encuentra el fomento del desarrollo sostenible (art. 3.5 y 21 TUE).

En este sentido, hay que destacar que la UE afirma mantener un fuerte compromiso con la realización de la Agenda 2030 a través del comercio internacional. Destacan la «Acción europea para la sostenibilidad» ${ }^{3}$ y la estrategia comercial adoptada en 2015 — «Comercio para todos»—, que contempla «una agenda de comercio que promueva el desarrollo sostenible, los derechos

2 Comisión Europea, Comercio para todos. Hacia una politica de comercio e inversión más responsable, 2015 , p. 5.

3 COM/2016/0739 final, de 22 de noviembre de 2016, Comunicación de la Comisión al Parlamento Europeo, al Consejo, al Comité Económico y Social Europeo y al Comité de las Regiones, sobre próximas etapas para un futuro europeo sostenible. Acción europea para la sostenibilidad. 
humanos y la buena gobernanza», de forma que se declara «la importancia de la posible contribución de la política comercial al desarrollo sostenible [que] se ha reafirmado recientemente en la Agenda 2030 para el Desarrollo Sostenible, que incluye los objetivos en este ámbito y guiará la actuación internacional en los próximos quince años» ${ }^{4}$.

Para lograr el objetivo de promocionar el desarrollo sostenible, desde la UE se ha fomentado la inclusión de disposiciones relativas a este fin en los tratados de libre comercio celebrados con terceros Estados, como son aquellos con Canadá, el Sistema de Integración Centroamericano, Colombia, Perú y Ecuador, Corea del Sur, Georgia, Japón, Mercosur, México, Moldavia, Singapur, Ucrania y Vietnam, y, al tiempo, se han desarrollado otras iniciativas relacionadas con la consecución de este objetivo, como son las evaluaciones de impacto de la sostenibilidad o la promoción del desarrollo sostenible y el buen gobierno mediante instrumentos preferenciales unilaterales. En este sentido, este trabajo tiene por objeto analizar la naturaleza del desarrollo sostenible en el marco de la Política Comercial Común (PCC), así como la formulación de los instrumentos que pretenden acometer este objetivo, de manera crítica y constructiva. No obstante, antes de ello hay que realizar una aproximación a cómo el desarrollo sostenible ha adquirido relevancia internacional, sobre todo tras la aprobación de la Agenda 2030 por parte de la Asamblea General de las Naciones Unidas en $2015^{5}$.

4 Comisión Europea, Comercio para todos..., cit. en nota 2, p. 21. Este documento sigue la estela trazada por los anteriores, como la labor desarrollada por el Parlamento Europeo en sus resoluciones, entre las que destacan la Resolución del Parlamento Europeo, de 25 de noviembre de 2010, sobre la política comercial internacional en el contexto de los imperativos del cambio climático (2010/2103(INI)) (2012/C 99 E/18), DO CE 99 de 3 de abril de 2012; la Resolución del Parlamento Europeo, de 25 de noviembre de 2010, sobre los derechos humanos y las normas sociales y medioambientales en los acuerdos comerciales internacionales (2009/2219(INI)) (2012/C 99/E07), DO CE 99 de 3 de abril de 2012; Resolución del Parlamento Europeo, de 25 de noviembre de 2010, sobre la responsabilidad social de las empresas en los acuerdos de comercio internacional (2009/2201(INI)) (2012/C 99 E/19) , DO CE 99 de 3de abril de 2012; o la Comunicación de la Comisión al Consejo, al Parlamento Europeo y al Comité Económico y Social Europeo, «Contribución al desarrollo sostenible: el papel del comercio justo y de los sistemas no gubernamentales de garantía de la sostenibilidad comercial», de 5 de mayo de 2009 (COM(2009)0215), que acaban desembocando en la Resolución del Parlamento Europeo, de 27 de septiembre de 2011, sobre una nueva política comercial para Europa en el marco de la Estrategia Europa 2020 (2010/2152(INI)) DO C 56 E de 26 de febrero 2013.

5 Asamblea General, Transformar el mundo: la Agenda 2030 para el Desarrollo Sostenible, A/RES/70/1, 21 de octubre de 2015. 


\section{EL DESARROLLO SOSTENIBLE Y EL DERECHO INTERNACIONAL. APROXIMACIÓN A LAS RELACIONES COMERCIALES}

El desarrollo sostenible, en su formulación original dada por la Comisión Brundtland, se concibe como la capacidad de que el desarrollo garantice y satisfaga las necesidades del presente sin comprometer la capacidad de que las generaciones futuras puedan satisfacer las suyas propias ${ }^{6}$. Esta definición arroja elementos económicos, sociales y medioambientales, los conocidos como tres pilares del desarrollo sostenible o retos, de siempre difícil articulación y concreción (Robinson y Tinker, 1997: 71). En este sentido, la Agenda 2030 supone un incuestionable avance, en la medida en que procede a establecer con enorme detalle en qué consiste la realización del desarrollo sostenible desde una perspectiva claramente finalista (Fernández Liesa, 2016: 81) mediante la formulación de los llamados Objetivos de Desarrollo Sostenible (ODS).

Ahora bien, cómo el desarrollo sostenible se enmarca en el derecho internacional no es ni ha sido una cuestión sencilla. Como ha señalado Rodrigo (2012: 134), el concepto de desarrollo sostenible ha podido ser considerado como un principio jurídico de naturaleza sustantiva o procedimental, como un concepto jurídico de naturaleza intersticial o incluso como un derecho humano. También se ha considerado un concepto con una clara vocación normativa, al tiempo que objetivo político, de lo que se deduce que parte de «un enfoque integral y cooperativo del desarrollo, ya que exige, a la vez, que se integren las dimensiones económica, social y medioambiental y que participen en su definición e implementación todos los actores afectados ya sean de naturaleza pública o privada» (Rodrigo, 2015:66). O, en palabras de Lowe, «a convenient [...] label for a general policy goal which may be adopted by states», incluso «a meta-principle, acting upon other legal rules and principles - a legal concept exercising a kind of interstitial normativity, pushing and pulling the boundaries of true primary norms when they threaten to overlap of conflict with each other» (Lowe, 1999: 31).

Se puede mantener que el desarrollo sostenible es un concepto puente, que aúna los ya aludidos tres aspectos en siempre complicado equilibrio —desarrollo económico, social y protección del medio ambiente- y que involucra a los países desarrollados y en desarrollo y a otros actores como empresas y sociedad civil en su realización. Como es sabido, la expresión

6 General Assembly, Development and International Economic Cooperation. Report of the World Commission on Environment and Development. Note by the Secretary-General. Annex. Report of the World Commission on Environment and Development «Our Common Future», A/42/427, 4 August 1987, apdo. 27. 
desarrollo sostenible ha formado parte de la agenda internacional desde el Informe Brundtland y desde entonces se le ha pretendido dotar de contenido, siendo para ello imprescindible aludir a las conferencias internacionales. Hay que tomar como punto de partida la Conferencia de Naciones Unidas sobre medio ambiente y desarrollo celebrada en Río de Janeiro en 1992, y continuar con la Cumbre sobre Desarrollo Sostenible de Johannesburgo de 2002, sin olvidar, como hito esencial, la Declaración del Milenio de 8 de septiembre de 2000 que establece los ocho objetivos de desarrollo del milenio (ODM). Los ODM sientan las bases de lo que quince años después será la Agenda 2030 (Díaz Barrado, 2016: 11), que prevé un marco de cooperación en el que los diferentes actores implicados tienen que seguir una serie de objetivos, esto es, un marco finalista (Fernández Liesa, 2016: 52-53), que constituyó una buena "combinación entre la necesidad de alcanzar metas concretas y la voluntad de dotar a la noción de desarrollo de los elementos que le permitan afirmarse como principio fundamental del orden internacional» (Díaz Barrado, 2016: 15).

Con la «Declaración de Nueva Delhi sobre los principios de derecho internacional relativos al desarrollo sostenible» de 2002 de la International Law Association ${ }^{7}$, la doctrina internacionalista pretende dotar de contenido a este principio, al tiempo que lo considera un objetivo global. En 2012, la Conferencia de Naciones Unidas sobre Desarrollo Sostenible (Río +20) sirve para establecer los componentes esenciales del concepto de desarrollo sostenible, conjugando nuevamente los aspectos económicos, sociales y ambientales, lo que acabará cristalizando en la Agenda 2030. Ahora bien, la inclusión del desarrollo sostenible en la Agenda 2030 podría servir para que el desarrollo sostenible se convierta en un principio estructural del orden internacional (Díaz Barrado, 2016: 8). Así también lo cree Bosselmann $(2016,92)$, que considera que la sostenibilidad es un principio esencial que influye en el orden internacional en su conjunto, no solo en las normas medioambientales. Ahora bien, la naturaleza del desarrollo sostenible en el orden internacional presenta complejas implicaciones, como indica Rodrigo, "debido a la incertidumbre existente sobre su valor político, jurídico y metodológico», aunque puede considerarse como «un concepto

7 World Summit on Sustainable Development, International New Delhi Declaration of Principles of International Law relating to Sustainable Development, Annex to the letter dated 6 August 2002 from the Permanent Representative of Bangladesh to the United Nations and the Chargé d'affaires a.i of the Permanent Mission of the Netherlands to the United Nations addressed to the Secretary-General of the United Nations, A/CONF.199/8, 9 August 2002. 
jurídico cuyo valor normativo puede tener pluralidad de manifestaciones y [...] puede ser concebido también como un marco metodológico para el análisis y cambio de la realidad por medio de la creación y aplicación de políticas públicas y normas jurídicas internacionales» (Rodrigo, 2015: 63). Siguiendo, pues, esta reflexión, y teniendo en cuenta la formulación de la Agenda 2030, el desarrollo sostenible se podría configurar como un objetivo a lograr por parte de toda la comunidad internacional, que implica a todos los Estados independientemente de su grado de desarrollo, y a todos los actores, públicos y privados, y cómo no, a la Unión Europea y a su política comercial. No obstante, ello no impide que el desarrollo sostenible hoy se presente como un concepto de «diferentes dimensiones" (Díaz Barrado, 2016: 18) o "pluralidad de manifestaciones» (Rodrigo, 2015: 69), dada la progresiva influencia que este término está adquiriendo.

Por lo que respecta a las relaciones comerciales, la Agenda 2030 alude a ellas en el ODS17, relativo a las alianzas; más concretamente, las metas 10, 11 y 12 se centran en ellas en los siguientes términos:

17.10 Promover un sistema de comercio multilateral universal, basado en normas, abierto, no discriminatorio y equitativo en el marco de la Organización Mundial del Comercio, incluso mediante la conclusión de las negociaciones en el marco del Programa de Doha para el Desarrollo;

17.11 Aumentar significativamente las exportaciones de los países en desarrollo, en particular con miras a duplicar la participación de los países menos adelantados en las exportaciones mundiales de aquí a 2020;

17.12 Lograr la consecución oportuna del acceso a los mercados libre de derechos y contingentes de manera duradera para todos los países menos adelantados, conforme a las decisiones de la Organización Mundial del Comercio, incluso velando por que las normas de origen preferenciales aplicables a las importaciones de los países menos adelantados sean transparentes y sencillas y contribuyan a facilitar el acceso a los mercados.

Varias son las cuestiones que nos interesan. La primera es la apuesta por el comercio multilateral. Que la Organización Mundial del Comercio está en una profunda crisis no es una novedad. Esta situación se deriva del bloqueo al que se ve sometida la Ronda de Doha, lo que se ha visto incrementado como consecuencia de la parálisis del mecanismo de solución de diferencias resultado de la postura obstruccionista de Estados Unidos en relación con la renovación de los miembros del órgano de apelación, a lo que se suma la sorprendente dimisión de su director general. Si bien la UE apoya formalmente el multilateralismo (véase la estrategia comercial «Comercio para todos» 
en su punto 5.1), no es menos cierto que la apuesta por el bilateralismo de la UE ha sido uno de los factores que han coadyuvado a la crisis del multilateralismo comercial (Antimiani y Salvatici, 2015: 253). Por lo que respecta al aumento de las exportaciones de los países en desarrollo, hay que señalar que no cabe duda de que el desmantelamiento arancelario que promueve la UE a través de los acuerdos bilaterales impulsa las exportaciones, aunque los acuerdos de naturaleza asimétrica pueden colocar en una situación de vulnerabilidad a los países en desarrollo (Valerdi, 2009: 12), especialmente grave para aquellos más débiles, como los países de África, Caribe y Pacífico (ACP), que podrían calificarse como los perdedores de la globalización (Gammage, 2017: 18). Este fenómeno no es querido ni deseado en el marco de la Agenda 2030, tal y como se ha podido ver en las metas del ODS17.

¿Son los tratados comerciales la única opción posible para promover las exportaciones de los países en desarrollo? Descartada la viabilidad de avances ciertos en la Ronda de Doha, parece que, desde el pragmatismo, la realidad nos lleva a plantearnos como el único escenario comercial posible el de las relaciones bilaterales, birregionales a lo sumo. Pero estas relaciones bilaterales no tienen que conducir necesariamente a la celebración de acuerdos de libre comercio. Como veremos más adelante, los esquemas preferenciales pueden servir también para promover las exportaciones al tiempo que las políticas sostenibles, lo que también podría servir a la tercera de las metas. Mediante el conocido esquema preferencial «Todo menos armas», que contempla la posibilidad de que los países menos avanzados (PMA) puedan exportar todos sus productos a la UE excepto armamento y munición libres de derechos de frontera, la Unión diseña su política comercial con este grupo de Estados. Ahora bien, en este esquema preferencial se plantea un problema relevante, y es el de la falta de capacidad de estos PMA para aprovechar las ventajas comerciales que proporciona, por lo que debe acompañarse de otros mecanismos de ayuda al comercio (Gradeva y Martínez-Zarzoso, 2010: 22), y ello a pesar de la flexibilidad que ha desarrollado la UE por lo que respecta a las reglas de origen, donde se contempla la acumulación bilateral, se prevén cuatro agrupaciones regionales en cuya virtud se permite la acumulación regional y la acumulación extendida para productos industriales y agrícolas procesados. Con todo, este esquema preferencial es una herramienta de indudable valor para promover las exportaciones originarias de estos Estados, tal y como se recuerda en la estrategia comercial «Comercio para todos» ${ }^{8}$.

8 Comisión Europea, Comercio para todos..., cit. en nota 2, p. 22). 


\section{LA NATURALEZA JURÍDICA DEL DESARROLLO SOSTENIBLE EN LA POLÍTICA COMERCIAL COMÚN}

En la UE hay que aludir al papel pionero desempeñado por el Parlamento Europeo", que ha facilitado la penetración del desarrollo sostenible en la PCC, dando voz a grupos de la sociedad civil y de interés, ya que hay que tener en cuenta que la PCC es la competencia exclusiva con mayor proyección de la UE, dado que los problemas comerciales lo son porque la UE únicamente puede ejercer su autoridad sobre otros actores a través de las relaciones comerciales, y es que es aquí donde podría cobrar fuerza el desarrollo sostenible, su formulación y su eficacia.

Ya se han señalado antes los esfuerzos realizados para dotar de contenido al concepto de desarrollo sostenible. Por lo que atañe a la UE, la aproximación al desarrollo sostenible no es exactamente igual a la ya vista, como se comprobará a continuación. El 26 de febrero de 2018 la Comisión publicó los quince puntos que debía desarrollar la acción de las instituciones europeas para implementar, reforzar y hacer más efectivos los capítulos sobre comercio y desarrollo sostenible contemplados en los acuerdos con terceros Estados, que atienden a los tres pilares en los que reposan los capítulos sobre desarrollo sostenible presentes en los acuerdos, como son «binding commitments by Parties to a range of multilateral environmental agreements and conventions of the International Labour Organization, structures to involve civil society organisations in the implementation of those commitments and a dedicated dispute settlement mechanism in which independent arbitrators make findings public of the fact regarding compliance» ${ }^{10}$.

Estos quince puntos tienen carácter cooperativo - colaboración con los Estados miembros y con el Parlamento; trabajo conjunto con organizaciones internacionales, como la Organización Internacional del Trabajo (OIT)—, se refieren a la participación de la sociedad civil — facilitación del monitoreo de la sociedad civil; extendiendo su participación a la totalidad de los acuerdos de libre comercio; supervisión de las actividades privadas-, a la entrega de resultados — valoración de las prioridades de los Estados; aplicación asertiva; fomento de la pronta ratificación de los acuerdos internacionales fundamentales; revisión de la efectividad de la implementación de los capítulos

9 Véase nota al pie número 3.

10 Comisión Europea, Feedback and way forward on improving the implementation and enforcement of Trade and Sustainable Development chapters in EU Free Trade Agreements, Non-paper of the Commission services, 2018. https://bit.ly/3epRLbD (último acceso: 31 de marzo de 2020). 
sobre desarrollo sostenible; elaboración de un manual sobre implementación; intensificación de recursos; acción por el clima; comercio y trabajo-, y a la transparencia y la comunicación — promoción de ambos; y plazos para dar respuestas a las solicitudes-. En definitiva, el desarrollo sostenible forma parte de la PCC, pero ¿cuál es su naturaleza jurídica?

El Tribunal de Justicia de la UE (TJUE) en su Dictamen 2/15 realiza un análisis de enorme interés sobre el lugar que ocupa el desarrollo sostenible en la PCC, en relación con el examen del tratado de libre comercio UE-Singapur, y las competencias que en él se regulan. Por lo que nos interesa, el TJUE analiza el capítulo 13, referido al desarrollo sostenible, en los apdos. 139 y siguientes.

Para el TJUE se trata de articular los objetivos que guían la acción exterior de la UE (arts. 3.5 y 21.2 TUE) con los objetivos de la PCC, de conformidad con lo establecido en el art. 207.1, que, en su segunda frase, establece que «la política comercial común se llevará a cabo en el marco de los principios y objetivos de la acción exterior de la Unión» ${ }^{11}$.

Estos principios y objetivos se contemplan en los arts. 21 y siguientes TUE. Más concretamente, el art. 21.2.f) alude a la contribución para «elaborar medidas internacionales de protección y mejora de la calidad del medio ambiente y de la gestión sostenible de los recursos naturales mundiales, para lograr el desarrollo sostenible». Como indica Beaucillon (2017: 822), el desarrollo sostenible se configura, pues, como un objetivo transversal que guía la acción exterior. Para su realización, la Unión se va a servir de sus políticas, incluida la PCC, lo que así reconoce el TJUE al afirmar con rotundidad en relación con la PCC: «La obligación de la Unión de integrar dichos objetivos y principios en la realización de la política comercial común». Ello «se deriva de una lectura conjunta del artículo 207 TFUE, apartado 1, segunda frase, del artículo 21 TUE, apartado 3, y del artículo 205 TFUE» ${ }^{12}$, lo que nos lleva a concluir que, en definitiva, «el objetivo de desarrollo sostenible forma parte integrante de la política comercial común $»^{13}$.

Esta interpretación es coherente con el impulso que la promoción del desarrollo sostenible ha tenido con el Tratado de Lisboa, en la medida en que la UE ha ampliado su marco competencial, por ejemplo, en materia de cooperación al desarrollo y ayuda humanitaria (art. 4.4. TFUE); la erradicación de la pobreza constituye uno de los objetivos de la UE en su acción exterior (arts.

11 Dictamen del Tribunal de Justicia, 2/15 de 16 de mayo de 2017, ECLI:EU:C:2017:376, apdo. 142 .

12 Dictamen 2/15, apdo. 143.

13 Dictamen 2/15, apdo. 147. 
208 TFUE y 3.5 TUE), y la cooperación al desarrollo ha sido reconocida como uno de los elementos de la acción exterior de la Unión (art. 21 TUE) (Olmos, 2016: 55).

Esta progresiva ampliación competencial es reconocida por el propio TJUE cuando afirma que

en lo concerniente a la política comercial común, el Tratado FUE difiere sensiblemente del Tratado CE, anteriormente en vigor, por cuanto incluye en dicha política nuevos aspectos del comercio internacional actual. La extensión del ámbito de la política comercial común por el Tratado FUE constituye una evolución significativa del derecho primario de la Unión (véase la sentencia de 18 de julio de 2013, Daiichi Sankyo y Sanofi-Aventis Deutschland, C-414/11, EU:C:2013:520, apartados 46 y 48$)^{14}$

lo que, como han afirmado Kleiman y Kübek (2017), confirma los «tectonic shifts» que el Tratado de Lisboa ha supuesto para la PCC.

Ahora bien, en principio los tratados de libre comercio no tienen como finalidad establecer los niveles de protección de las cuestiones objeto de la promoción del desarrollo sostenible — básicamente derechos laborales y protección medioambiental—, sino regular las relaciones comerciales. Y aquí es donde el TJUE realiza su principal aportación (Castillo de la Torre, 2018: 494) en relación con el encuadre del desarrollo sostenible en la PCC. Como indica Segura Serrano (2017: 127), el Tribunal alude al vínculo específico del desarrollo sostenible con el comercio entre las partes del acuerdo ${ }^{15}$, en la medida en que el capítulo 13 «regula ese comercio garantizando que se realice dentro del respeto a esos convenios y que ninguna medida adoptada en virtud de éstos se aplique a dicho comercio de un modo que constituya una discriminación arbitraria o injustificable o una restricción encubierta ${ }^{16}$. $\mathrm{Y}$, además, procede a «desconectar la PCC de las competencias internas en el ámbito de la protección laboral y medioambiental» (Segura Serrano, 2017:128).

Es más, el TJUE llega a afirmar que

la especificidad del vínculo que presentan las disposiciones del capítulo 13 del Acuerdo proyectado con el comercio entre la Unión y la República de Singapur se deriva también de que una infracción de las disposiciones en materia de protección social de los trabajadores y de protección del medio ambiente, que figuran en ese capítulo, autoriza a la otra Parte a finalizar o suspender la liberalización de ese comercio prevista en las demás disposiciones del Acuerdo, conforme a la norma

14 Dictamen 2/15, apartado 141.

15 Ibid., apdo. 155.

16 Ibid., apdo. 156. 
consuetudinaria de Derecho internacional codificada en el artículo 60, apartado 1, de la Convención sobre el Derecho de los Tratados, firmada en Viena el 23 de mayo de $1969^{17}$.

Esta afirmación no es menor, y es que, como recuerda Ankersmit (2017), el incumplimiento de las disposiciones de este capítulo podría llegar a constituir una violación de un elemento esencial del acuerdo — «ha de considerarse -dice el TJUE- que este capítulo 13 ocupa un lugar esencial en el acuerdo» ${ }^{18}$ — y que da lugar a una cierta condicionalidad en tanto que supedita la liberalización comercial al respeto de las obligaciones en desarrollo sostenible $^{19}$, lo que recuerda a la práctica de la condicionalidad en materia de derechos humanos, aunque no llega a tener su alcance ya que el acuerdo no recoge ninguna cláusula de este tipo.

Ello no impide, no obstante, que se pueda afirmar que para el TJUE el desarrollo sostenible es un objetivo de la Unión que puede realizarse a través de la PCC, e, incluso, se considere como un objetivo de la propia PCC, en tanto que forma parte integrante de ella ${ }^{20}$. El que se valore al desarrollo sostenible como objetivo - lo que ya hizo Rodrigo al considerarlo como un objetivo político (2015: 65) — puede tener importantes implicaciones también jurídicas, como por ejemplo a la hora de «elaborar o reorientar las políticas económicas, sociales y medioambientales» (Rodrigo, 2015:67).

\section{IV. ¿CÓMO SE PREVÉ LA PROMOCIÓN DEL DESARROLLO SOSTENIBLE EN LA RELACIÓN COMERCIAL CON TERCEROS ESTADOS?}

Las previsiones sobre desarrollo sostenible han adquirido tres formas distintas (Zvelc, 2009: 174). Así, la más habitual ha sido la inserción de disposiciones a través de capítulos en los tratados de libre comercio celebrados con terceros Estados. Aparte de los capítulos sobre desarrollo sostenible, la UE ha venido implementado las evaluaciones de impacto sobre la sostenibilidad (conocidas como SIAs, en sus siglas en inglés). Las SIAs tienen por objeto examinar los efectos que los tratados de libre comercio pueden tener en las partes, y se realizan con carácter previo a la conclusión del tratado. Por otro lado, creo oportuno añadir a los sistemas de preferencias generalizadas de la

\footnotetext{
Ibid., apdo. 161.

Ibid., apdo. 162.

9 Ibid., apdo. 166.

Ibid., apdo. 147.
} 
UE, más concretamente al SPG+, que mediante la condicionalidad positiva promueve el desarrollo sostenible con una serie de $\operatorname{Estados}^{21}$. Se dedicarán las siguientes páginas al análisis de estos tres elementos.

\section{LOS CAPÍTULOS SOBRE DESARROLLO SOSTENIBLE}

La primera vez que el objetivo de promover el desarrollo sostenible se contempló en un acuerdo con terceros Estados fue en el Acuerdo de Cotonú con los países de $\mathrm{ACP}^{22}$, lo que se vio reflejado en el acuerdo de partenariado económico con el Cariforum en $2008^{23}$. Posteriormente, este camino fue seguido en el acuerdo con Corea del $\mathrm{Sur}^{24}$, primero en contemplar un capítulo sobre desarrollo sostenible, lo que ha continuado en los acuerdos con el Sistema Centroamericano de Integración (SICA) ${ }^{25}$, con Moldavia ${ }^{26}$, Georgia $^{27}$, Ucrania ${ }^{28}$, Colombia y Perú ${ }^{29}$, al que después se sumó Ecuador ${ }^{30}$, con Canadá ${ }^{31}$, Singapur ${ }^{32}$ y Japón ${ }^{33}$. La forma en la cual se han contemplado estas disposiciones ha cambiado. Así, como ha indicado Lowe, las primeras aproximaciones a la inclusión del desarrollo sostenible en los acuerdos comerciales consistieron en la inclusión de normas en cuya virtud las partes se comprometían a respetar las obligaciones contenidas en otros acuerdos internacionales, como el Acuerdo de París, o los convenios de la OIT. No obstante, los acuerdos más recientes modifican el enfoque. Así, el Acuerdo Económico y Comercial Global celebrado con Canadá (conocido como CETA, por sus siglas en inglés) incluye tres capítulos, uno sobre comercio y desarrollo sostenible, otro sobre comercio y trabajo, y uno más sobre comercio y medio ambiente (Lowe, 2019), aunque, como recuerda Harrison (2019: 711), si bien existen

21 Reglamento (UE) n. ${ }^{\circ}$ 978/2012 del Parlamento Europeo y del Consejo de 25 de octubre de 2012, sobre el esquema generalizado de preferencias (DO L272, de 16 de octubre de 2015).

22 DO L 317 de 15 de diciembre de 2000.

23 DO L 289, de 30 de octubre de 2008.

24 DO L 127, de 14 de mayo de 2011.

25 DO L 346, de 15 de diciembre de 2012.

26 DO L 260, de 30 de agosto de 2014.

27 DO L 261, de 30 de agosto de 2014.

28 DO L 161, de 29 de mayo de 2014.

29 DO L 354, de 21 de diciembre de 2012.

30 DO L 356, de 24 de diciembre de 2016.

31 DO L 11 de 14 de enero de 2017.

32 DO L 294, de 14 de noviembre de 2019.

33 DO L 330, de 27 de diciembre de 2018. 
una ciertas diferencias entre los capítulos de desarrollo sostenible contenidos en los tratados del libre comercio con terceros, hay elementos centrales que se encuentran en todos ellos, en la medida en que es posible identificar la misma aproximación al contexto, a las normas aludidas, a los mecanismos de monitoreo y a la forma de gestionar los disensos.

No cabe duda de que estos capítulos responden a la promoción del desarrollo sostenible, y su virtualidad reside en la incorporación de elementos no comerciales a la relación entre las partes en el tratado. Esta relación va a gozar de mecanismos de supervisión. Así, van a crear un órgano colegiado encargado de realizar un seguimiento (Comité de Comercio y Desarrollo Sostenible), y órganos consultivos nacionales, conformados por organizaciones del Estado y un mecanismo de diálogo con la sociedad civil. Estos mecanismos sirven para resolver las dudas que puedan aparecer a lo largo de la vida del instrumento convencional en relación con el cumplimiento de las disposiciones sobre desarrollo sostenible. Llegado el caso de que existan dudas sobre la aplicación de estos capítulos, puede designarse un grupo de expertos que conozca de la cuestión. Pero hay que señalar que es cierto que estos capítulos están al margen de los mecanismos de solución de controversias contemplados en los tratados para cuestiones comerciales, y carecen de elementos de coerción similares. De hecho, ello ha conducido a que haya autores que han señalado que la aproximación de la UE a estas cuestiones sea calificable de soft approach (Olmos, 2016: 50), y es que otros actores internacionales dan calidad jurídica a estos ámbitos, como hace Estados Unidos en sus relaciones con terceros Estados, que llega a prever la imposición de sanciones en caso de incumplimiento (Horn, Mavroidis y Sapir, 2009: 16), como se ha podido comprobar en relación con los incumplimientos cometidos por Guatemala (Hradilová y Svoboda, 2018: 1036). Este carácter blando del enfoque europeo viene a ser corroborado por la redacción de estas disposiciones, que adolecen de falta de normatividad y se caracterizan por su carácter programático (Adriaensen y González-Garibay, 2013: 543). Harrison (2019: 713-714) destaca otros defectos, como el que los funcionarios europeos desconozcan la existencia de determinados problemas laborales en los socios, la falta de atención a los problemas laborales dentro de la UE, la cooperación entre las partes que no se encuentra implementada de forma sistemática, y que cuando surgen controversias, las disposiciones blandas resultan inadecuadas, dada la falta de coerción, lo que tiene un efecto disuasorio limitado.

Por lo que atañe al contenido de estos capítulos, como ya se ha indicado, dos son los ámbitos objeto de regulación: estándares laborales y medioambientales. En los primeros, y tomando como referencia el tratado con Corea del Sur, «se reafirma el compromiso, en virtud de la Declaración Ministerial de 2006 del Consejo Económico y Social de las Naciones 
Unidas sobre el Empleo Pleno y el Trabajo Decente, a reconocer el empleo pleno y productivo y el trabajo decente para todos como elementos clave del desarrollo sostenible para todos los países» (art. 13.4.2). En consecuencia, se van a incluir los cuatro estándares laborales referentes a derechos fundamentales, a saber, «libertad de asociación y reconocimiento efectivo del derecho a la negociación colectiva; eliminación de todas las formas de trabajo forzado u obligatorio; abolición efectiva del trabajo infantil; y eliminación de la discriminación en materia de empleo y ocupación». Así, las partes se obligan a implementar los convenios OIT ratificados y a esforzarse por seguir ratificando aquellos que no lo estén, lo que ha dado lugar a tensiones entre las partes, como se verá más adelante.

Por otro lado, se reconoce la relevancia de los acuerdos medioambientales multilaterales, que se comprometen a fomentar la adhesión a los tratados más relevantes, así como su implementación, intentando alcanzar niveles altos de protección ${ }^{34}$. Ahora bien, como indican Hradilová y Svoboda (2018: 1024), estas disposiciones van más allá e incluyen cuestiones como el manejo sostenible de los recursos naturales, el comercio de bienes y servicios sostenibles que faciliten la transición ecológica, el etiquetado de productos sostenibles, el tráfico ilegal de especies en peligro, o la pesca ilegal, no declarada y no reglamentada.

Llegados aquí conviene plantearse si el enfoque propiciado por la UE es el adecuado. Raess (2018) ha llevado a cabo un análisis sobre esta cuestión que considero oportuno traer a colación, ya que, mediante la utilización de indicadores, ha concluido que la inclusión de estas disposiciones en los tratados, acompañados de mecanismos de cooperación, está asociada con una reducción de las violaciones in law de los derechos laborales en los países en desarrollo, esto es, en una mejora del marco jurídico. Ahora bien, no se puede decir lo mismo, de forma general, de las violaciones in practice.

Por lo que respecta a la efectividad de las disposiciones medioambientales, Raess (2018) señala que hay menos estudios, aunque se puede concluir que el enfoque suave adoptado por la UE tiene menos efectos en la protección medioambiental que aquellos modelos más coercitivos, como el desarrollado por Estados Unidos desde 1994 en el acuerdo con México y Canadá. Ahora bien, siguiendo a Hradilová y Svoboda (2018: 1040), tampoco parece que el modelo estadounidense sea la panacea, y apuestan por un reforzamiento del diálogo y la cooperación, así como de la participación de la sociedad civil. En mi opinión, y dada la práctica de la Unión Europea con la condicionalidad

34 «Environment and Trade and External Relations», European Commission, https:// bit.ly/2APJnUn (último acceso: 30 de marzo de 2020). 
instaurada a través de la cláusula democrática, pareciera que estos capítulos están en un segundo nivel por lo que atañe a su normatividad (Bartels, 2014: 33). Y es que, como señalan Luyten y Woo Kim (2020), en la UE no existen ni las normas ni los mecanismos necesarios para tomar medidas que impliquen la suspensión del acuerdo en caso de incumplimiento de los capítulos sobre desarrollo sostenible. Además, como señala Harrison (2019: 721), el enfoque debería replantearse, para situar el desarrollo sostenible en el corazón de los acuerdos comerciales de la UE, dado que el desarrollo sostenible está encerrado ("ghettoised» en la redacción original en inglés) en capítulos aparte, en lugar de contemplarse como una cuestión transversal, relevante para todos los aspectos del acuerdo comercial.

\section{LOS SIAS}

Las evaluaciones de impacto de la sostenibilidad (SIAs, por sus siglas en inglés), son una herramienta de la Dirección General de Comercio de la Comisión Europea que pretenden analizar los impactos que los tratados de libre comercio van a tener tanto en la UE como en el tercer Estado con el que se negocia el acuerdo.

La UE ha sido un adalid de estas evaluaciones de impacto, ya que propuso incluirlas en la Ronda del Milenio de la Organización Mundial del Comercio $^{35}$. Esta filosofía ha sido desarrollada con éxito por la UE, que, dada la proliferación de acuerdos con terceros Estados, las SIAs han pasado a ser una herramienta clave de su labor negociadora. Así, en 2002, en las negociaciones del acuerdo de libre comercio con Chile se implementa por primera vez un SIA, al que han seguido los hechos con los Estados árabes del Golfo (2004), con los países ACP (2007), el Área Euromediterránea de libre comercio (2007), Ucrania (2007), Corea del Sur (2008), China (2008), Mercosur (2009), India (2009), ASEAN (2009), Centroamérica (2009), Libia (2009), Comunidad Andina de Naciones (2009), Canadá (2011), Georgia y Moldavia (2012), Armenia (2013), Marruecos y Túnez (2013) Egipto y Jordania (2014), Japón (2016), Myanmar (2016), bienes ecológicos (2016), Estados Unidos (2017), comercio de servicios (2017) y China (2018). Hoy en día se están llevando a cabo evaluaciones en las negociaciones con Australia y Nueva Zelanda, en la modernización de los acuerdos

35 «Sustainability Impact Assessment of Proposed WTO New Round of Multilateral Trade Negotiations", European Commission, https://bit.ly/3fKTMiO (último acceso: 28 de marzo de 2020). 
con Chile y México, en las negociaciones con Indonesia, Filipinas y Malasia, $y$ en las inacabables con Mercosur.

Dos ediciones del «Manual sobre las SIAs» ha publicado la Comisión, la primera en 2006 y la segunda en 2016, que fue objeto de una consulta pública y que es la que actualmente establece su metodología. De acuerdo con este instrumento, las SIAs son evaluaciones ex ante, llevadas a cabo por consultores independientes que analizan los cambios que el acuerdo comercial puede llegar a causar, identifican posibles ámbitos de compensación e intentan que las mejores opciones sean las escogidas. Así, se lleva a cabo un serio análisis del potencial económico, social, de derechos humanos y medio ambiental, tanto para la UE como para los socios comerciales, incluso para otros Estados relevantes, en el marco de un proceso de consulta continuo y amplio, transparente, en el que se involucra a todas las partes interesadas ${ }^{36}$.

Si bien a priori parece que los SIAs son una herramienta valiosa, han recibido críticas por parte de la doctrina. Como bien indica Douma (2017: 208), no acaba de estar claro que sus recomendaciones se sigan en la práctica, incluso algunas de las inquietudes que han planteado han sido subestimadas, y es que, en ocasiones, su publicación tardía ha tenido como consecuencia que lo avanzado de las negociaciones impidiera que fueran tenidas en cuenta. Una crítica que en mi opinión puede añadirse a las SIAs es que la evaluación únicamente se realiza ex ante y no ex post, lo que podría ser conveniente para conocer de qué manera el acuerdo ha impactado en el tercer Estado o en la UE, o incluso en otros Estados. Dado el importante número de acuerdos celebrados con países en desarrollo, considero que estas evaluaciones podrían ser de enorme utilidad de cara a conocer cómo el acuerdo ha repercutido en el ámbito comercial, pero también en las relaciones laborales y en la preservación del medio ambiente.

\section{EL SPG+}

De los tres esquemas de preferencias comerciales unilaterales de la UE, solo el Sistema de Preferencias Generalizadas Plus (SPG+) tiene como objetivo primordial la promoción del desarrollo sostenible. Este régimen preferencial, denominado formalmente como «régimen especial de estímulo del desarrollo sostenible y la gobernanza», se regula en el Reglamento Delegado (UE) n. ${ }^{\circ}$

36 Comisión Europea, Handbook for trade sustainability impact assessment, segunda edición, Bruselas, 2016, p. 8. 


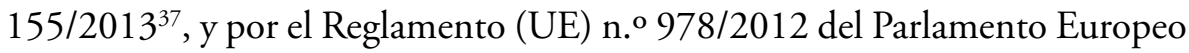
y del Consejo ${ }^{38}$.

Según el art. 9 del reglamento de 2012, el SPG+ tendrá como Estados beneficiarios a los considerados vulnerables, según la definición del anexo VII del reglamento de 2012, que no dispongan de otro tipo de relación comercial con la UE, esto es, que no sean parte en un tratado comercial, y que hayan ratificado una serie de convenios internacionales en relación con los cuales no conste ningún incumplimiento grave por parte de los órganos de supervisión. Los tratados internacionales aludidos son los relativos a la protección y promoción de los derechos humanos en el ámbito universal ${ }^{39}$, convenios de la OIT relativos al respeto de los derechos de los trabajadores ${ }^{40}$, los instrumentos convencionales más emblemáticos en relación con

37 Reglamento Delegado (UE) n. ${ }^{\circ}$ 155/2013 de la Comisión de 18 de diciembre de 2012 por el que se establecen normas relativas a los procedimientos de concesión del régimen especial de estímulo del desarrollo sostenible y la gobernanza con arreglo al

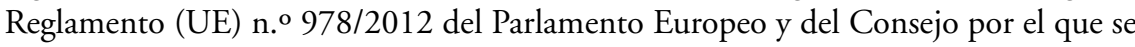
aplica un sistema de preferencias arancelarias generalizadas, DO L 48, de 21 de febrero de 2013.

Reglamento (UE) n.o 978/2012 del Parlamento Europeo y del Consejo de 25 de octubre de 2012 por el que se aplica un sistema de preferencias arancelarias generalizadas y se deroga el Reglamento (CE) n. ${ }^{\circ}$ 732/2008 del Consejo, DO L 303, de 31 de octubre de 2012.

39 Los instrumentos aludidos son: Convención para la Prevención y la Sanción del Delito de Genocidio (1948), Convención Internacional sobre la Eliminación de todas las Formas de Discriminación Racial (1965), Pacto Internacional de Derechos Civiles y Políticos (1966), Pacto Internacional de Derechos Económicos Sociales y Culturales (1966), Convención sobre la Eliminación de todas las Formas de Discriminación contra la Mujer (1979), Convención contra la Tortura y Otros Tratos o Penas Crueles, Inhumanos o Degradantes (1984) y Convención sobre los Derechos del Niño (1989).

40 Convenio sobre el Trabajo Forzoso, n. ${ }^{\circ} 29$ (1930), Convenio sobre la Libertad Sindical y la Protección del Derecho de Sindicación, n. ${ }^{o} 87$ (1948), Convenio sobre el Derecho de Sindicación y de Negociación Colectiva, n. 98 (1949), Convenio relativo a la Igualdad de Remuneración entre la Mano de Obra Masculina y la Mano de Obra Femenina por un Trabajo de Igual Valor, n. ${ }^{o} 100$ (1951), Convenio sobre la Abolición del Trabajo Forzoso, n. ${ }^{\circ} 105$ (1957), Convenio relativo a la Discriminación en Materia de Empleo y Ocupación, n. ${ }^{\circ} 111$ (1958), Convenio sobre la Edad Mínima, n. ${ }^{\circ} 138$ (1973) y Convenio sobre la Prohibición de las Peores Formas de Trabajo Infantil y la Acción Inmediata para su Eliminación, n. ${ }^{\circ}$ 182 (1999). 
la preservación y protección del medio ambiente ${ }^{41}$ y buen gobierno ${ }^{42}$ (anexo VIII).

Los Estados beneficiarios del SPG+ —Cabo Verde, Armenia, Kirguistán, Mongolia, Pakistán, Filipinas, Sri Lanka, Bolivia y Paraguay- disfrutan de una completa eliminación de los derechos en frontera para más del $66 \%$ de las líneas arancelarias de productos, y se someten a la supervisión por parte de la UE del cumplimiento continuado de las condiciones que les han hecho beneficiarios de este esquema preferencial.

Dos son, por tanto, las características del SPG+. La primera, que es un instrumento subsidiario de los tratados de libre comercio, esto es, una vez que un Estado ha celebrado un acuerdo con la UE, dejará de ser beneficiario, lo cual también plantea dudas acerca de cuál es el régimen más favorable para un país en desarrollo teniendo en cuenta la asimetría de la relación entre las partes (Manger y Shadlen, 2014: 80). La segunda se deriva de la consideración de este instrumento como un mecanismo de condicionalidad positiva, que comparte la perspectiva del desarrollo sostenible mantenida en otros instrumentos — disposiciones relativas a la preservación del medio ambiente y normas de protección social- como son los capítulos de desarrollo sostenible previstos en los acuerdos con terceros Estados, pero también enfoques más novedosos, como los ya vistos en las SIAs, con la introducción de los derechos humanos como parte de un concepto más avanzado de desarrollo sostenible, y que es coherente con la mantenida en la Agenda 2030.

Llegados aquí, conviene señalar que contamos con una miríada de normas recogidas en diversos acuerdos, así como técnicas de indudable interés, como las SIAs, que tienen como objeto servir a la promoción del desarrollo sostenible en los términos previstos en los tratados, e instrumentos que se caracterizan por una técnica avanzada de condicionalidad positiva. Ahora bien, ¿se adecúan estas prácticas a la Agenda 2030?

41 Convención sobre el Comercio Internacional de Especies Amenazadas de Fauna y Flora Silvestres (1973), Protocolo de Montreal relativo a las Sustancias que agotan la Capa de Ozono (1987), Convenio de Basilea sobre el Control de los Movimientos Transfronterizos de los Desechos Peligrosos y su Eliminación (1989), Convenio sobre la Diversidad Biológica (1992), Convención Marco de las Naciones Unidas sobre el Cambio Climático (1992), Protocolo de Cartagena sobre Seguridad de la Biotecnología (2000) y Convenio de Estocolmo sobre Contaminantes Orgánicos Persistentes (2001).

42 Convención Única de las Naciones Unidas sobre Estupefacientes (1961), Convenio de las Naciones Unidas sobre Sustancias Psicotrópicas (1971), Convención de las Naciones Unidas contra el Tráfico Ilícito de Estupefacientes y Sustancias Psicotrópicas (1988) y Convención de las Naciones Unidas contra la Corrupción (2004). 


\section{LA ADECUACIÓN DE LOS INSTRUMENTOS COMERCIALES EUROPEOS A LA AGENDA 2030}

Al examinar la adecuación de la PCC a la Agenda 2030 se pueden distinguir dos perspectivas. La primera, y de espectro general, se refiere a la toma en consideración de las tres vertientes que conforman el concepto de desarrollo sostenible. Los capítulos incluidos en los tratados contemplan disposiciones relativas a protección medioambiental y normas laborales, y se enmarcan en acuerdos que disfrutan de una importante proyección económica, dado que su objetivo es la liberalización de lo esencial de los intercambios comerciales entre las partes, con la finalidad de fomentar el crecimiento económico. Así, los tres pilares del desarrollo sostenible están presentes, aunque la visión más amplia otorgada al concepto por la Agenda 2030 no lo está. Esta visión clásica del concepto de desarrollo sostenible es compartida también por el SPG+, si bien es cierto que este instrumento parte de una visión más amplia que los capítulos, al incluir el cumplimiento de instrumentos relativos a la protección de los derechos humanos y de buen gobierno, por lo que se aproxima más a lo establecido en la Agenda. Y lo mismo se puede decir de las SIAs, que analizan cuestiones de orden económico, medioambiental y social, pero que desde 2012 también incluyen la evaluación del impacto relativa a los derechos humanos. De todas formas, puede afirmarse que la perspectiva amplia y finalista otorgada al desarrollo sostenible a través de la Agenda 2030 no es compartida plenamente por los instrumentos comerciales europeos. Los capítulos sobre desarrollo sostenible mantienen una visión tradicional del concepto, por lo que quedan lejos de la aproximación más avanzada. Por su parte, las SIAs y el SPG+, si bien parten de un enfoque más próximo, aún no participan de la visión omnicomprensiva que proporcionan los ODS.

La segunda perspectiva nos reconduce al análisis realizado más arriba en relación con las metas que aluden a cuestiones comerciales, en el ODS17, relativo a las alianzas. Como ya se ha dicho, probablemente la manera en que la UE está integrando el desarrollo sostenible en su política comercial no sea la que mejor se adecúe a la Agenda 2030. Además de lo ya indicado en relación con la asimetría de las relaciones comerciales y las implicaciones que ello tiene para los países en desarrollo, la preferencia por los tratados comerciales en lugar de los esquemas preferenciales los está convirtiendo en subsidiarios. Ello no impide que volvamos sobre otra cuestión que considero preocupante como son las carencias normativas de los capítulos sobre desarrollo sostenible. El carácter programático de estos capítulos ya ha sido indicado y ello conduce a situar en un segundo plano la relevancia de estas materias en las relaciones de las partes. Existe un debate en la Unión acerca de cómo mejorar estos 
capítulos, pero no hay consenso sobre cómo desarrollar esta labor ${ }^{43}$. Ahora bien, se están realizando avances en este ámbito que pueden poner en cuestión el soft approach de la UE. Prueba de ello son dos procedimientos iniciados por la UE, uno de los cuales versa específicamente sobre capítulos de desarrollo sostenible y otro, aunque no lo hace concretamente en este ámbito, está claramente conectado. Comencemos por este último. El 20 de junio de 2019 la delegación de la UE en Ucrania solicitó el establecimiento de un panel de arbitraje en relación con las restricciones a la exportación de madera sin procesar, al considerar que estas restricciones no están en conformidad con el acuerdo de asociación, que las prohíbe ${ }^{44}$. Este procedimiento versa sobre restricciones cuantitativas, prohibidas ya en el marco multilateral (art. XI GATT de 1994), obligación que incorpora el acuerdo de asociación a través de su art. 35. En este sentido, Ucrania podría basar su postura en el art. XX. b) del GATT —-medidas «necesarias para [...] preservar los vegetales»—o XX.g) — medidas «relativas a la conservación de los recursos naturales agotables»—, incorporados a través del art. 36 del acuerdo, aunque no parece sencillo que las medidas ucranias se adecúen al chapeau del art. XX, esto es, que no "constituyan un medio de discriminación arbitrario o injustificable [...] o una restricción encubierta al comercio internacional». Con todo, es cierto que estamos ante una controversia que afecta a una cuestión de índole medioambiental, y que, tal vez, pueda arrojar luz sobre el necesario equilibrio entre la liberalización comercial y la preservación de los recursos naturales (Romanchyshyna, 2019).

Por otro lado, es preciso aludir al procedimiento abierto en el marco del art. 13 del acuerdo con Corea del Sur. En diciembre de 2018 la UE solicitó consultas ${ }^{45}$ a Corea del Sur en relación con determinadas medidas vigentes en el país asiático que limitaban los derechos laborales, entre ellas la sindicación, así como la falta de compromiso en relación con la ratificación de distintos convenios de la OIT. Estas consultas fracasaron, por lo que la UE solicitó el establecimiento de un panel de expertos que sirviera para conocer si Corea del Sur cumple con sus compromisos en el marco del capítulo sobre desarrollo sostenible. Dicho panel de expertos fue creado

43 Comisión Europea, Feedback and way forward on improving the implementation and enforcement of Trade and Sustainable Development chapters in EU Free Trade Agreements, Non-paper of the Commission services, 2018. https://bit.ly/2YnFsqt (último acceso: 31 de marzo de 2020).

44 European Union. Delegation to Ukraine, Note Verbale, Ref. Ares(2019) 392926920/06/2019. https://bit.ly/37YX8fy (último acceso: 2 de junio de 2020).

45 Republic of Korea- Compliance with obligations under Chapter 13 of the EU-Korea Free Trade Agreement. Request for the establishment of a Panel of Experts by the European Union, 4 July 2019. 
el 19 de diciembre de $2019^{46}$. No cabe duda de que este procedimiento despierta un profundo interés, y que su resultado puede tener consecuencias muy importantes en el futuro de los capítulos sobre desarrollo sostenible. En mi opinión, el camino debería dirigirse hacia una mejor calidad normativa (Harrison et al., 2019: 273), y aunque genera incertidumbre la aproximación coercitiva (Hradilova y Svoboda, 2018: 1039), no cabe duda de la necesidad de mejorar su técnica, tal vez apostando por la transversalidad del desarrollo sostenible, como se ha indicado antes.

Los tratados de libre comercio no son la única herramienta disponible. Tal vez un mayor protagonismo de los esquemas preferenciales, que podrían ser examinados ex ante y ex post a través de las SIAs, pudiera ser oportuno. La Agenda 2030 no exige la apertura comercial bilateral entre Estados, plantea la necesidad de reforzar el marco multilateral —que contempla a través de la cláusula de habilitación las preferencias comerciales unilaterales-, y la ampliación de la capacidad exportadora de los países en desarrollo y de los PMA. En mi opinión, el ODS17 y sus metas comerciales respaldan la labor desempeñada por los sistemas preferenciales. En consecuencia, no parece que relegarlos a un segundo plano sirva para promover la Agenda 2030.

\section{CONCLUSIÓN}

El desarrollo sostenible es un objetivo de la PCC. Son tres las modalidades seguidas por la UE para promover el desarrollo sostenible: los capítulos sobre desarrollo sostenible previstos en los tratados de libre comercio, las SIAs como herramienta de evaluación y el mecanismo preferencial condicional: el SPG+.

Por otro lado, la Agenda 2030, en su objetivo 17, relativo a las alianzas, concreta tres metas que afectan a las relaciones comerciales y que, en síntesis, se basan en una apuesta clara por el multilateralismo comercial representado por la Organización Mundial del Comercio, en el refuerzo de la capacidad exportadora de los países en desarrollo y en la mejora de las capacidades de los PMA. En mi opinión, estos marcos pueden coincidir en una mayor presencia de los esquemas preferenciales y del modelo que representa el SPG+, que, hoy, no es sino residual de la relación convencional bilateral. Además, las SIAs

46 Procedural information related to EU-Korea dispute settlement on Labour, Brussels, 19 December 2019. 
podrían también aplicarse a los esquemas preferenciales, lo que permitiría tener una visión más amplia de su impacto, tanto ex ante como ex post.

Por lo que respecta a la relación convencional bilateral, en ocasiones birregional, para ser adaptada a las prioridades establecidas en la Agenda 2030 debe ser, al menos, modulada, impulsando los mecanismos preferenciales y reforzando los capítulos en los acuerdos. Las SIAs, por su parte, son un elemento clave, que permitirá constatar de qué manera las relaciones comerciales de la UE sirven al objetivo de promover el desarrollo sostenible, sobre todo si fueran realizadas también ex post, al tiempo que su utilización podría extenderse a los mecanismos preferenciales para aclarar sus efectos.

\section{Bibliografía}

Adriaensen, J. y González-Garibay, M. (2013). The Illusion of Choice: The European Union and the Trade-Labor Linkage. Journal of Contemporary Research, 9 (4), 542-559.

Ankersmit, L. (2017). Opinion 2/15: Adding some spice to the trade and environment debate. European Law Blog [blog], 15-06-2017. Disponible en: https:// bit.ly/2YES7V1.

Antimiani, A. y Salvatici, L. (2015). Regionalism versus Multilateralism: The case of the European Union Trade Policy. Journal of World Trade, 49 (2), 253-276.

Bartels, L. (2014). A Model Human Rights Clause for the EU's International Trade Agreements. Berlin: German Institute for Human Rights.

Beaucillon, C. (2017). Opinion 2/15: Sustainable is the New Trade. Rethinking Coherence for the New Common Commercial Policy. European Papers, 2 (3), 819-828. Disponible en: http://doi.org/10.15166/2499-8249/177.

Bosselman, K. (2016). The Principle of Sustainability: Transforming law and governance. London: Routledge. Disponible en: https://doi.org/10.4324/9781315553962.

Castillo de la Torre, F. (2018). El Tribunal de Justicia y las relaciones exteriores tras el Tratado de Lisboa. Revista de Derecho Comunitario Europeo, 60, 491-512. Disponible en: https://doi.org/10.18042/cepc/rdce.60.01.

Díaz Barrado, C. (2016). Los objetivos de desarrollo sostenible: un principio de naturaleza incierta y varias dimensiones fragmentadas. Anuario Español de Derecho Internacional, 32, 9-48. Disponible en: https://doi.org/10.15581/010.32.9-48.

Douma, W. T. (2017). The Promotion of Sustainable Development through EU Trade Instruments. European Business Law Review, 28 (2), 197-216.

Fernández Liesa, C. R. (2016). Transformaciones del Derecho internacional por los objetivos de desarrollo sostenible. Anuario Español de Derecho Internacional, 32, 49-81. Disponible en: https://doi.org/10.15581/010.32.49-81.

Gammage, C. (2017). North-South Regional Trade Agreements as LegalRegimes. London: Edward Elgar. Disponible en: https://doi.org/10.4337/9781784719623. 
Gradeva, K. y Martínez-Zarzoso, I. (2010). The Role of the Everything but Arms Trade Preferences Regime in the EU Development Strategy. Hannover: German Development Economics Conference.

Harrison, J. (2019). The Labour Rights Agenda in Free Trade Agreements. Journal of World Investment and Trade, 20 (5), 705-725. Disponible en: https://doi. org/10.1163/22119000-12340153.

Harrison, J., Barbu, M., Campling, L., Richardson, B. y Smith, A. (2019). Governing Labour Standards through Free Trade Agreements: Limits of the European Union's Trade and Sustainable Development Chapters. Journal of Common Market Studies, 57 (2), 260-277. Disponible en: https://doi.org/10.1111/ jcms. 12715 .

Horn, H., Mavroidis, P. C. y Sapir, A. (2009). Beyond the WTO? An Anatomy of EU and US Preferential Trade Agreements. Brussels: Bruegel. Disponible en: https:// doi.org/10.2139/ssrn.1411066.

Hradilova, K. y Svoboda, O. (2018). Sustainable Development Chapters in the EU Free Trade Agreements: Searching for Effectiveness. Journal of World Trade, 52 (6), 1019-1042.

Kleiman, D. y Kübek, G. (2017). The Singapore Opinion or the End of Mixity as We Know It. Verfassungsblog [blog], 23-05-2017. Disponible en: https://bit. ly/2AIlgGO.

Lowe, S. (2019). The EU should reconsider its approach to trade and sustainable development. Centre for European Reform-Insights, 31-10-2019. Disponible en: https://doi.org/10.1093/acprof:oso/9780198298076.003.0002.

Lowe, V. (1999). Sustainable Development and Unsustainable Arguments. En A. Boyle y D. Freestone (ed.). International Law and Sustainable Development. Past Achivements and Future Challenges (pp. 19-37). Oxford: Oxford University Press.

Luyten, E. y Woo Kim, J. (2020). Could the EU's Chief Trade Enforcement Officer enforce sustainable development commitments under EU trade agreements against non-compliant third countries? EULawLive, 13-05-2020. Disponible en: https:// bit.ly/3ftBz9e.

Mälstrom, C. (2016). Foreword a Handbook for trade sustainability impact assessment. Brussels: European Commission.

Manger, M. S. y Shadlen, K. C. (2014). Political Trade Dependence and NorthSouth Trade Agreements. International Studies Quarterly, 58 (1), 79-91. Disponible en: https://doi.org/10.1111/isqu.12048.

Olmos, B. (2016). Squaring the Circle Balancing Sustainable Development and Investment Protection in the EU Investment Policy. European Energy and Environmental Law Review, 25 (2), 44-55.

Raess, D. (2018). Labour (and environmental) provisions in FTAs: What do they do? En Workshop: The future of sustainable development chapters in EU free trade agreements. European Parliament.

Robinson, J. y Tinker, J. (1997). Reconciling Ecological, Economic and Social Imperatives: A New Conceptual Framework. En T. Schrecker (ed.). The Social and 
Environmental Dimensions (pp. 71-94). London: Palgrave Macmillan. Disponible en: https://doi.org/10.1007/978-1-349-25648-8_5.

Rodrigo, Á. (2012). El principio de integración de los aspectos económicos, sociales y medioambientales del desarrollo sostenible. Revista Española de Derecho Internacional, 64 (2), 133-161.

- (2015). El desafío del desarrollo sostenible. Madrid: Marcial Pons.

Romanchyshyna, I. (2019). Guest Post: Brussels Demands Wood: A Precedent of Resorting to FTA Arbitration under the EU-Ukraine Association Agreement. International Economic Law and Policy Blog [blog], 04-07-2019. Disponible en: https://bit.ly/2C3ZnSG.

Segura Serrano, A. (2017). Renovación y crisis de la Política Comercial Común de la Unión Europea: el Dictamen 2/15. Revista General de Derecho Europeo, 43, 100-151.

Valerdi Rodríguez, E. (2009). Los tratados de libre comercio de la Unión Europea: implicaciones para los paises en desarrollo. Documento de trabajo; 8/2009. Real Instituto Elcano. Disponible en: https://bit.ly/37zSrIO.

Zvelc, R. (2009). Environmental integration in EU trade policy: The generalised system of preferences, trade sustainability impact assessments and free trade agreements. En E. Morguera (ed.). The External Environmental Policy of the European Union. EU and International Law Perspectives (pp. 174-203). Cambridge: Cambridge University Press. Disponible en: https://doi.org/10.1017/CBO9781139152327.011. 
